\title{
Study of the Behavior of Different Guidewire Shapes in a Patient-specific Numerical Model for Transcatheter Aortic Valve Implantation
}

\author{
Phuoc Vy ${ }^{1,2,3,4}$, Vincent Auffret ${ }^{3,4,5}$, Miguel Castro ${ }^{3,4},{\text { Pierre } \text { Badel }^{2} \text {, Michel Rochette }}^{1}$, Pascal \\ Haigron $^{3,4}$, Stéphane Avril ${ }^{2}$, Hervé Le Breton ${ }^{3,4,5}$ \\ ${ }^{1}$ ANSYS France, Villeurbanne, France \\ ${ }^{2}$ Ecole Nationale Supérieure des Mines de Saint-Etienne, CIS-EMSE, INSERM:U1059, \\ SAINBIOSE, Saint-Etienne, France \\ ${ }^{3}$ INSERM, U1099, Rennes, France \\ ${ }^{4}$ LTSI, Université de Rennes 1, Rennes, France \\ ${ }^{5}$ CHU Rennes, Service de Cardiologie et Maladies Vasculaires, Rennes
}

\begin{abstract}
Transcatheter Aortic Valve Implantation is a miniinvasive procedure to replace aortic valves. The approach consists in delivering a fully collapsible bioprosthesis to the native valve site through a catheter. Stiff guidewires are required to deliver, stabilize and deploy the prosthesis by fitting in the left ventricular apex but they can also damage the ventricular wall of the patient. Nowadays, guidewires of different sizes, stiffness and shapes are available. In order to find the most appropriate guidewire for each patient, modelling could provide a better understanding of the interactions between the guidewire and the patient's left ventricle walls. Our objective was to explore the influence of guidewire shape on the contact conditions using numerical simulations.

We have developed a Finite Element model to simulate the stiff guidewire inserted into the left ventricle. The model was solved using the ANSYS software with an implicit resolution scheme. We explored different shapes for the distal end of the guidewire. We observed the distribution of the contact forces. An adequate curvature size resulted in smaller maximum pressure.
\end{abstract}

\section{Introduction}

Aortic stenosis (AS) is a widespread cardiovascular disease with increasing incidence in aging population, affecting almost $10 \%$ of octogenarians [1]. Symptoms of severe AS include dyspnea, chest pain, syncope, acute congestive heart failure, and sudden death. Severe symptomatic AS requires timely aortic valve replacement. Transcatheter Aortic Valve Implantation (TAVI) is a mini-invasive procedure to replace aortic valves and showed similar results as surgical treatment among intermediate or high surgical-risk patients [2-4]. The most common approach for TAVI is the femoral artery. Using a dedicated catheter, a stiff guidewire is inserted from the femoral artery to the left ventricle to assist the navigation of the prosthesis along the aortic arch. The fully crimped prosthesis is delivered by a catheter which glides over the guidewire to reach the aortic valve.

To provide support to the delivery catheter, the tip of the stiff guidewire lies against the ventricular wall, which can lead to left ventricular perforation if too much pressure is applied on the guidewire or if the shape of the guidewire does not suit the left ventricle. However, subtle manipulations of the guidewire allow fine adjustments of the prosthesis position before its deployment and are essential to the success of the procedure. Nevertheless, surgeons have a limited perception on the intra-operative behaviour of the guidewire. The most suitable guidewire shape and the amount of pressure that can be safely applied to this guidewire remain unclear in clinical practice.

The understanding of the behaviour of the tools appears as a key to better control the procedure. Studying the interactions between the guidewire and the ventricular wall could help choose an appropriate guidewire shape to decrease the risk of ventricular perforation. Several simulation studies have already simulated prosthesis deployment within the patient-specific aortic valve [5]. These numerical models seemed in agreement with the decision of the medical team for the simulated patient cases. However, to the best of our knowledge, there is no numerical study about the relationship between guidewire shape and its interaction with the ventricle. To address this lack, a contact analysis was performed on two retrospective patient cases using numerical simulation of guidewire insertion. 


\section{Materials and methods}

\subsection{Geometries}

Images were retrospectively collected after the procedure of two patients (noted case A and B) who underwent TAVI in the cardiovascular department of the Rennes University Hospital. An intra-operative angiography was acquired during the protocol (Figure 1a) but the precise shape of the guidewire pre-deformed by the practitioner was unknown. A pre-operative CT-scan was segmented to extract the thoracic aorta and the left ventricle. The geometry was smoothed and imported in ANSYS for numerical simulation (Figure 1b).

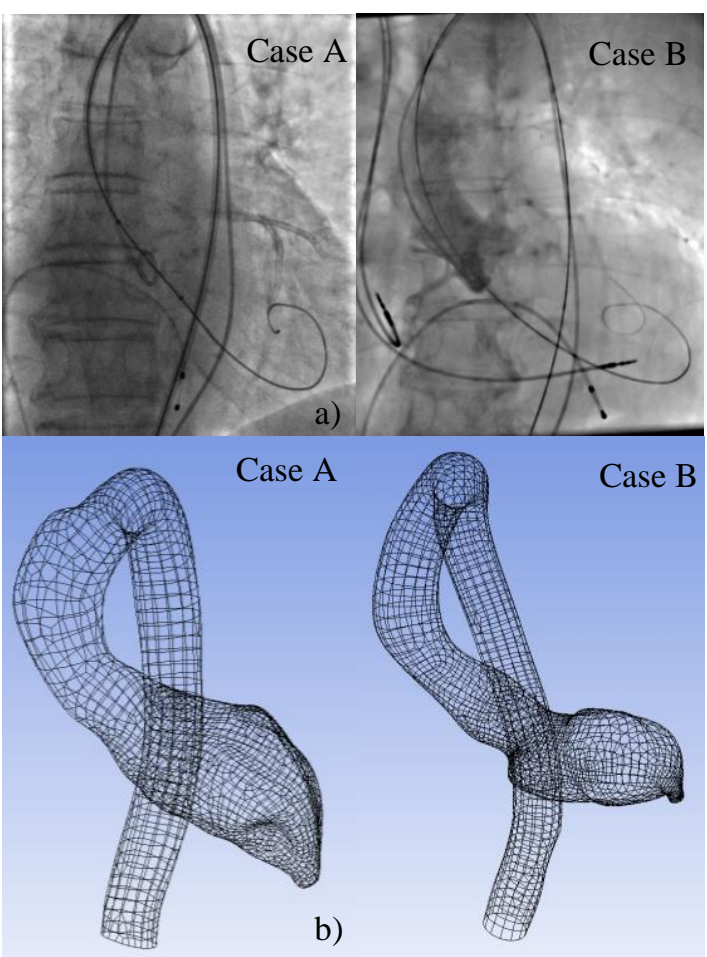

Figure 1. Patient-specific geometry of patient case A (left) and patient case B (right) a) intra-operative angiography b) simulation mesh.

The influence of the shape of the guidewire predeformation was studied by varying its radius of curvature from $15 \mathrm{~mm}$ to $30 \mathrm{~mm}$ (Figure $2 \mathrm{a}$ ).

\subsection{Assumptions}

The CT image showed a fully dilated ventricle at diastole. The guidewire did not induce noticeable deformation of the aortic and ventricular walls according to our observations of the angiography. Thus, they were assumed rigid. The insertion of the guidewire in the ventricle was assumed to be quasi-static because the contact forces outweighed the inertia effects. Friction forces were neglected because the guidewire had a PTFE coating designed to reduce friction. It was assumed that deformation of the guidewire far from the ventricle marginally impacted the result of the study.

a)

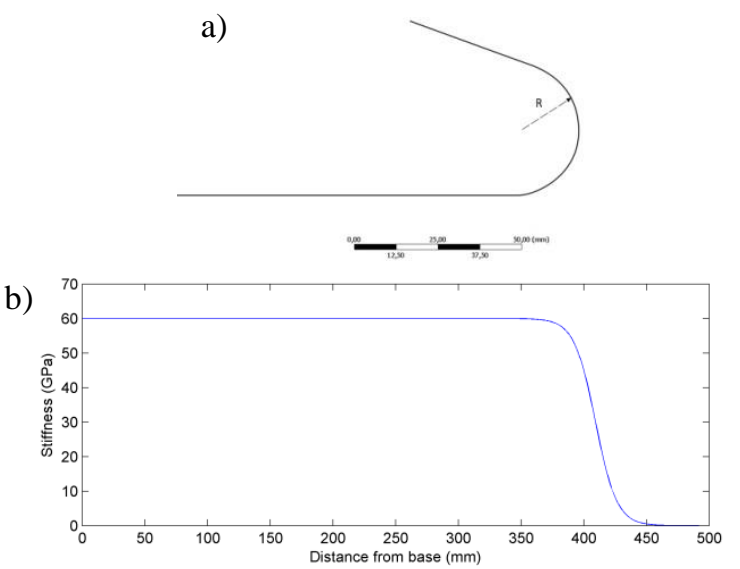

Figure 2. Numerical stiff guidewire shape a) geometry b) stiffness of the guidewire.

\subsection{Numerical model}

The numerical model was defined on the commercial software ANSYS 17.1. The simulations were performed with full Newton-Raphson method and sparse matrix direct solver.

The geometry was meshed in ANSYS. The patient mesh included 8341 nodes and 2794 contact element. The guidewire mesh had 4165 nodes and 1388 elements. The average distance between the nodes was $0.2 \mathrm{~mm}$. The patient mesh was rigid and the guidewire behaviour was modelled with a linear elastic model. The stiffness profile of the guidewire is given in Figure $2 b$.

The guidewire was maintained tangential to the centerline at the exit point of the femoral sheath used for tools insertion in the abdominal aorta. Its longitudinal displacement was constrained and the corresponding insertion force was evaluated. Contact was numerically enforced using Augmented Lagrangian method. The simulation results were compared to intra-operative views in order to qualitatively assess the accuracy of the simulation.

\subsection{Contact analysis}

The contact analysis concerned the region of the guidewire inserted in the ventricle (Figure 3). The nodal contact force along the region of interest was plotted. The maximum contact force was qualitatively compared among the contact zones. The general reaction force of the ventricle acting on the guidewire was evaluated by the vector sum of the nodal forces. 

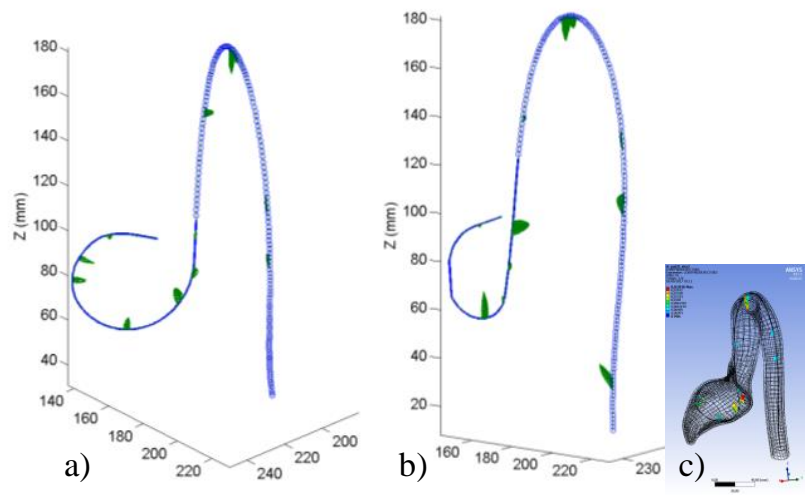

guidewire body

region of interest for contact analysis nodal contact force

Figure 3. Side view of guidewire insertion in the ventricle with an insertion force of $0.20 \mathrm{~N}$ a) large curvature (30 $\mathrm{mm})$ b) small curvature $(15 \mathrm{~mm}) \mathrm{c}$ ) overview of the mesh orientation.

\section{Results}

\subsection{Comparison to intra-operative data}

The pre-operative geometries were registered on the intra-operative angiography by matching the contour of the contrast-injected aortic root. The intra-operative guidewire curvature was unknown but simulated guidewires with a $30 \mathrm{~mm}$ radius showed satisfying match in the region of interest (Figure 4).

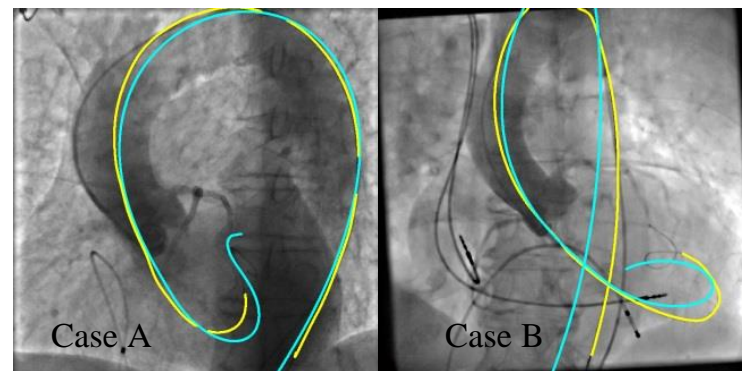

Figure 4. Projection of simulated guidewires (cyan) on intra-operative guidewires (yellow).

\subsection{Contact results}

Figure 5 shows the comparison between a small and a large curvature (noted R15 and R30 for $15 \mathrm{~mm}$ and 30 $\mathrm{mm}$ ), as well as the comparison of a low and high insertion force (noted F0.10 and F0.20 for $0.10 \mathrm{~N}$ and $0.20 \mathrm{~N}$ ) on patient case A. Figure 6 shows the comparison of the curvature size on patient case B at an insertion force of $0.20 \mathrm{~N}$.

In case A with F0.10, the larger curvature had a higher maximum force than the small curvature near the aortic valve. However, it did decrease the contact force applied on the ventricular wall from $0.018 \mathrm{~N}$ to $0.007 \mathrm{~N}$ (around $375 \mathrm{~mm}$ on guidewire length). This effect can also be observed in Figure 3, at the bottom of the curvature. The larger curvature increased the number of contact zone along the guidewire (from 5 to 8 in case A, from 3 to 8 in case B). Case B shows a decrease of maximum nodal force from $0.025 \mathrm{~N}$ down to $0.019 \mathrm{~N}$ by using a larger curvature. The length of the guidewire in contact increased from $72.8 \mathrm{~mm}$ to $94.9 \mathrm{~mm}$. The sum of the norm of the contact force remained $0.16 \mathrm{~N}$.

Increasing the insertion force resulted in different behavior according to the curvature size (Figure 7). The vector sum increased for small curvature. This suggested that the contact forces were more directional. This is supported by an increased reaction force at the ventricular wall resulting from higher insertion force (Figure $5 b$ around $380 \mathrm{~mm}$ ). In the larger curvature case, insertion resulted in higher contact force at the tip of the guidewire and in a shifting of the contact zone toward the left of Figure 5a. In other word, the guidewire slid on the wall with a coiling movement as opposed to the small curved guidewire, which was simply pushing deeper in the ventricular wall.

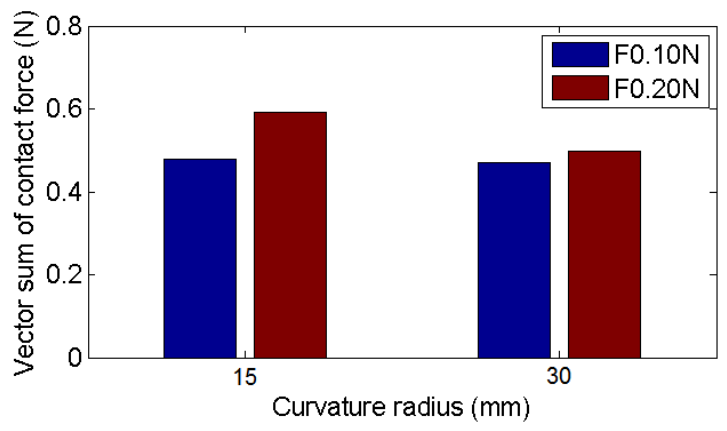

Figure 7. Norm of vector sum of the contact nodal force in the ventricle for case A.

\section{Conclusion}

This study outlined qualitative trends describing the behavior of a stiff guidewire in the left ventricle. The largest curvature size for the guidewire shape provided a sliding effect which limited the magnitude of contact force on the ventricular wall. In the opposite, a small curvature increased the contact force in a concentrated area on the ventricular wall.

Numerical model could be used in a patient-specific method to determine the safest guidewire shape. Our preliminary results have to be confirmed by further studies. They should address the issues related to material properties of the patient's vascular structures, the range of curvature sizes of the guidewire and the validation on a representative set of cases. 

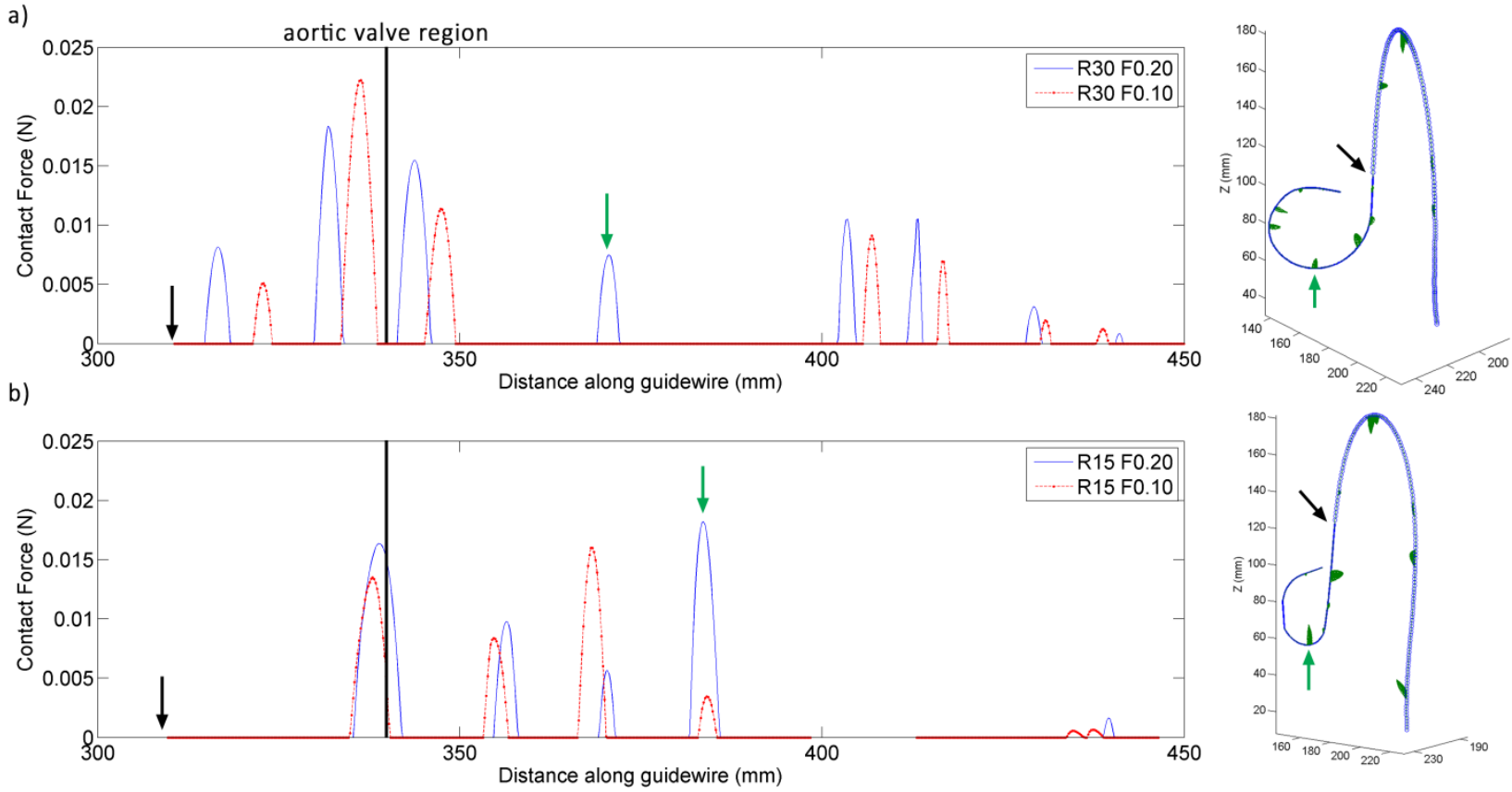

Figure 5. Contact forces along the guidewire in case $\mathrm{A}$ with an insertion force of $0.10 \mathrm{~N}$ and $0.20 \mathrm{~N}$ for a) large curvature $(30 \mathrm{~mm})$ and b) small curvature $(15 \mathrm{~mm})$. A $3 \mathrm{D}$ view of the simulated guidewire is shown in the right. Arrows on the graph are reflected back on the 3D view.

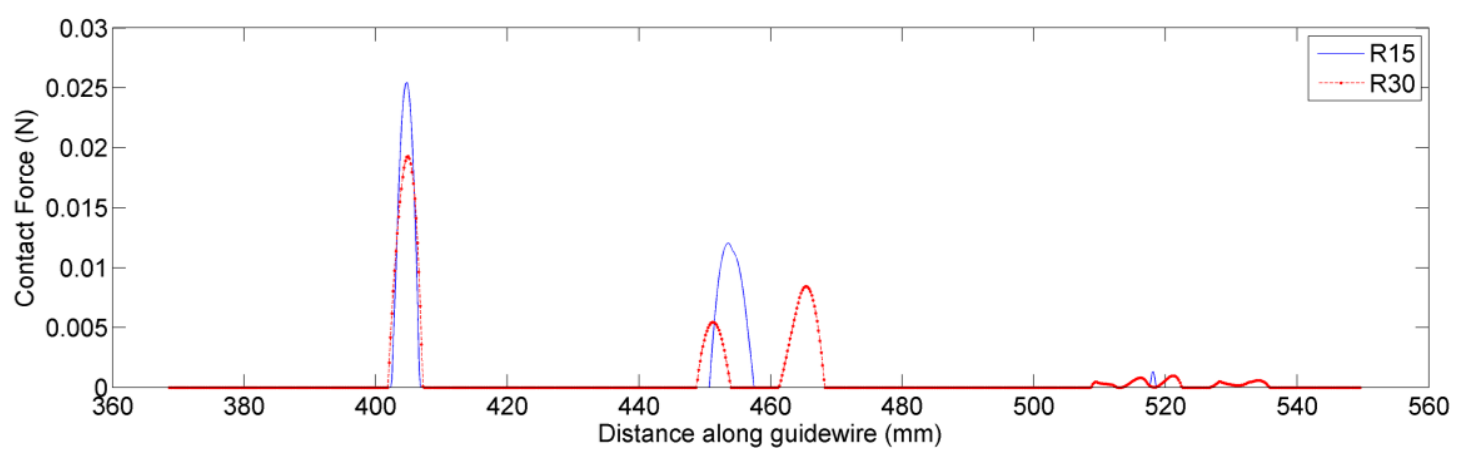

Figure 6. Contact forces along the guidewire in case B with an insertion force $0.20 \mathrm{~N}$ for large curvature $(30 \mathrm{~mm})$ and small curvature $(15 \mathrm{~mm})$.

\section{Acknowledgements}

This work has been conducted in the experimental platform TherA-Image (Rennes, France) supported by Europe FEDER.

\section{References}

[1] Eveborn GW, Schirmer H, Heggelund G, Lunde P, Rasmussen K. The evolving epidemiology of valvular aortic stenosis. The Tromso Study. Heart 2012;99:396-400.

[2] Leon MB et al. Transcatheter or Surgical Aortic-Valve Replacement in Intermediate-Risk patients. N Engl J Med 2016;374:1609-20.

[3] Siontis GCM et al. Transcatheter aortic valve implantation vs. surgical aortic valve replacement for treatment of severe aortic stenosis: a meta-analysis of randomized trials. European Heart Journal 2016;37:3503-12.
[4] Reardon MJ et al. Surgical or Transcatheter Aortic-Valve Replacement in Intermediate-Risk Patients. N Engl J Med 2017;376:1321-31.

[5] Wang Q et al. Simulations of transcatheter aortic valve implantation: implications for aortic root rupture. Biomech. Model. Mechanobiol. 2015;14:29-38.

Address for correspondence.

Phuoc VY

CIC-IT de Rennes

CHU de Rennes

Centre cardio pneumologique

Rue Henri Le Guilloux

35033 Rennes

phuoc.vy@ansys.com 